\title{
A linear-time algorithm for the orbit problem over cyclic groups
}

\author{
Anthony W. Lin · Sanming Zhou
}

Received: date / Accepted: date

\begin{abstract}
The orbit problem is at the heart of symmetry reduction methods for model checking concurrent systems. It asks whether two given configurations in a concurrent system (represented as finite strings over some finite alphabet) are in the same orbit with respect to a given finite permutation group (represented by their generators) acting on this set of configurations by permuting indices. It is known that the problem is in general as hard as the graph isomorphism problem, whose precise complexity (whether it is solvable in polynomial-time) is a long-standing open problem. In this paper, we consider the restriction of the orbit problem when the permutation group is cyclic (i.e. generated by a single permutation), an important restriction of the problem. It is known that this subproblem is solvable in polynomial-time. Our main result is a linear-time algorithm for this subproblem.
\end{abstract}

Keywords symmetry reductions $\cdot$ model checking $\cdot$ cyclic groups $\cdot$ orbits

\section{Introduction}

Since the inception of model checking (cf. [9]), a key challenge in verifying concurrent systems has always been how to circumvent the state explosion problem, which is exponential in the number of processes and in the number of finite-domain variables. The fundamental algorithmic problem can essentially be construed as a reachability problem in an exponentially-sized graph that is succinctly represented (e.g. in some concurrent programming language). Among others, symmetry reduction [20,11,16] has emerged to be an effective technique in combatting the state

A. W. Lin

Yale-NUS College

10 College Ave West, Singapore 138609

Tel.: +65 6601-3699

E-mail: anthony.w.lin@yale-nus.edu.sg

S. Zhou

School of Mathematics and Statistics

University of Melbourne

Parkville, Victoria 3010, Australia. 
explosion problem. The essence of symmetry reduction is to identify symmetries in the system and avoid exploring states that are "similar" (under these symmetries) to previously explored states, thereby speeding up model checking.

Every symmetry reduction method has to deal with the following two computationally difficult problems: (1) how to identify symmetries in the given system, and (2) how to check that two configurations are similar under these symmetries. To simplify our discussion of Problem 1, we will restrict our discussion to process symmetries. [Extensions to data symmetries are possible, e.g., see the recent result of [28], which gives a general reduction of process and data symmetry identification in concurrent systems to symmetry identification in the solutions to constraints in the constraint-satisfaction problem.] In this case, for concurrent systems with $n$ processes, Problem 1 amounts to searching for a group $G$ of permutations on $[n]:=\{1, \ldots, n\}$ such that the system behaves in an identical way under the action of permuting the indices of the processes by any $\pi \in G$. For example, for a distributed protocol with a ring topology, the group $G$ could be a rotation group generated by the "cyclical right shift" permutation RS that maps $i \mapsto i+1 \bmod n$ for each $i \in[n]$. See Example 1 for concrete examples. Although Problem 1 is computationally hard in general, a lot of research advances has been made in the past decade (e.g. see the recent survey [27, and also the recent paper 28 for a more general technique that covers both process and data symmetries). Now the group $G$ partitions the state space of the concurrent system (i.e. $\Gamma^{n}$ for some finite set $\Gamma$ ) into equivalence classes called $(G$-)orbits. Problem 2 is essentially the orbit problem (over finite permutation groups): given $G$ and two configurations $\mathbf{v}, \mathbf{w} \in \Gamma^{n}$, determine whether $\mathbf{v}$ and $\mathbf{w}$ are in the same $G$-orbit. For example, if $G$ is generated by $\operatorname{RS}$ with $n=4$, the two configurations $(1,1,0,0)$ and $(0,0,1,1)$ are in the same orbit. These two computational problems can be studied independently. The focus of this paper is the second problem, i.e., the orbit problem.

Example 1 Two token-passing protocols with multiple tokens: These examples are nondeterministic versions of the randomised self-stablising protocol of Israeli and Jalfon [21] (also see [24]).
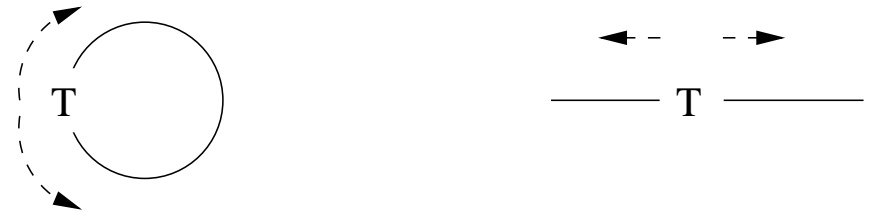

In the first example (left figure), there are $n$ processes $P_{1}, \ldots, P_{n}$ connected in a ring-shaped topology (i.e. the neighbours of $P_{i}$ are $P_{i+1} \bmod n$ and $\left.P_{i-1} \bmod n\right)$. There are $m \leq n$ tokens in the network, each held by a unique process. At any given step, a unique process $P_{i}$ holding a token is nondeterministically chosen by a scheduler and is permitted to pass the token to its neighbour $P_{j}$ (i.e. either the left $P_{i-1} \bmod n$ neighbour or the right neightbour $P_{i+1} \bmod n$ ). If $P_{j}$ already had a token, it will simply merge the two tokens into one, which reduces the total number of tokens in the network by 1 . For 
each number $n$ of processes, this description yields a transition system. For example, configurations of the system are of the form $\left(\alpha_{1}, \ldots, \alpha_{n}\right) \in\{\perp, \top\}^{n}$, where $\top($ resp. $\perp$ ) constitutes that the process holds (resp. does not hold) a token. The symmetry group $G_{n}$ of the transition system is the Dihedral group $D_{n}$, which is generated by the cyclical right shift $\mathrm{RS}(i \mapsto i+1 \bmod n)$ and the reflection $(i \mapsto n-i$, for each $i \in\{1, \ldots, n\})$. In the standard composition of disjoint cycles notation, these permutations can be written as $(1,2, \ldots, n)$ and $(1, n)(2, n-1) \cdots(\lfloor n / 2\rfloor,\lceil n / 2\rceil)$, respectively.

In the second example (right figure above), we modify the first example by disconnecting the line between $P_{1}$ and $P_{n}$, which results in a line-shaped topology. In effect, $P_{1}$ (resp. $P_{n}$ ) can only pass a token to $P_{2}$ (resp. $P_{n-1}$ ). The symmetry group $G_{n}^{\prime}$ of the system in this case is the group generated by the reflection mapping that maps $i \mapsto n-i$, for each $i \in\{1, \ldots, n\}$.

The orbit problem (OP) was first studied in the context of model checking by Clarke et al. [11 in which it was shown to be in NP but is as hard as the graph isomorphism problem, whose precise complexity (whether it is solvable in polynomial-time) is a long-standing open problem. The difficulty of the problem is due to the fact that the input group $G$ is represented by a set $S$ of generators and that the size of $G$ can be exponential in $|S|$ in the worst case. There is also a closely related variant of $\mathrm{OP}$ called the constructive orbit problem $(C O P)$, which asks to compute the lexicographically smallest element $\mathbf{w} \in \Gamma^{n}$ in the orbit of a given configuration $\mathbf{v} \in \Gamma^{n}$ with respect to a given group $G$. OP is easily reducible to $\mathrm{COP}$, though the reverse direction is by no means clear. COP was initially studied in the context of graph canonisation by Babai and Luks 2, in which COP was shown to be NP-hard (in contrast, OP is unlikely to be NP-hard since it would imply the collapse of the polynomial-time hierarchy to its second leve 1 ). In the context of model checking, COP was first studied by Clarke et al. [10, in which a number of "easy groups" for which COP becomes solvable in P are given including polynomial-sized groups (e.g. rotation groups), the full symmetry group $\mathcal{S}_{n}$ (i.e. containing all permutations on $[n])$, and disjoint/wreath products of easy groups (cf. [15]).

In this paper, we consider the orbit problem over cyclic groups (i.e. generated by a single permutation $\pi \in \mathcal{S}_{n}$ ), which is an important subproblem of OP. In the case of rotation groups, one can do a simple enumeration of the group elements and solve the orbit problem in polynomial-time. [More precisely, if the group has $m$ elements, this algorithm runs in time $O(m n)$, which is already quadratic over rotation groups.] However, cyclic subgroups of $\mathcal{S}_{n}$ can even be of size exponential in $n$ (see Proposition 3 below), which rules out this enumeration strategy. It turns out that the orbit problem over cyclic groups is known to be solvable in polynomialtime (e.g. see 2, 23, where this is shown for a much larger class of permutation

\footnotetext{
1 For, if it were NP-hard, then the coset intersection problem (for permutation groups) would be NP-hard, owing to its polynomial-time equivalence to the orbit problem [10]. By the well-known results of 3.17 (also see [18. Section 6.5, Chapter 27]), which essentially shows that the coset intersection problem is in the complexity class COAM (contained in the second level of the polynomial hierarchy), this would mean that the polynomial hierarchy collapses to the second level.
} 
groups denoted as $\Gamma_{d}$ for every fixed $d$, which contains solvable groups). Another way to see that OP over cyclic groups is solvable in polynomial-time is by a quadratic-time reduction to the classical orbit problem over rational matrices [22]: given a rational $n$-by- $n$ matrix $M$ and two rational vectors $\mathbf{v}, \mathbf{w} \in \mathbb{Q}^{n}$, determine if there exists $k \in \mathbb{N}$ such that $M^{k} \mathbf{v}=\mathbf{w}$. In fact, the two problems coincide when $M$ is restricted to permutation matrices [7, i.e., 0-1 matrices with precisely one column for each row with entry 1 . That OP over cyclic groups is in $\mathrm{P}$ follows from Kannan and Lipton's celebrated result [22] that OP over rational matrices is in P.

Mere polynomial time-complexity is far from sufficient for the purpose of symmetry reduction methods, since a model checker will have to invoke an algorithm for the orbit problem once each time a new configuration in the given transition system is visited (e.g. see [27]). Recent case studies in 28 suggest that the cost of solving the orbit problem often becomes extremely prohibitive, even more so than the cost of computing the symmetries2. Therefore, lightweight methods for dealing with the orbit problem are crucial for the success of symmetry reductions in model checking.

Contributions. In this paper, we provide an algorithm for the orbit problem over cyclic groups that runs in linear-time. To this end, we provide a linear-time reduction to the problem of solvability of systems of linear congruence equations. The reduction exploits subtle connections to the string searching problem.

As for the solvability of systems of linear congruence equations, there is a well-known algorithm (based on the extended Euclidean algorithm) that runs in linear-time assuming constant-time integer arithmetic operations. However, when we measure the number of bit operations (i.e. bit complexity model), it turns out that the algorithm runs in time cubic in the number of equations in the systems. To address this issue, we restrict the problem to input instances provided by our reduction from the orbit problem. We offer two solutions. Firstly, we show that the average-case complexity of the algorithm under the bit complexity model is $O\left(\log ^{5} n\right)$, which is sublinear [Here, $n$ measures the size of the input to the orbit problem.] Secondly, we provide another algorithm that uses at most linearly many bit operations in the worst case (though on average it is worse than the first algorithm).

It turns out that permutation groups generated by two permutations already suffice to make the orbit problem as hard as the graph isomorphism problem. This is almost a direct corollary of the polynomial-time reduction in [1] from the graph isomorphism problem to the orbit problem over some group $G$. It turns out the group $G$ that is produced by the reduction of [11] is not any arbitrary group and could easily be generated by two generators (for the same reason that the full symmetry group $\mathcal{S}_{n}$ on $\{1, \ldots, n\}$ can be generated by the permutations $(1,2)$ and $(1,2, \ldots, n))$.

Organisation. Section 2 contains basic definitions, notations, and results that will be used throughout the rest of the paper. We provide our linear-time reduction from the orbit problem to equations solving in Section 3 (Algorithm 2). Thus far, we assume that arithmetic operations take constant time. We deal with the issue of bit complexity in Section 4. We conclude with future work in Section 5 .

2 Some examples in 28] (even with a small number of processes) require a model checker to invoke an algorithm for the orbit problem hundreds to thousands of times for one transition system. 
Acknowledgment. We thank the anonymous referees of the conference version for their helpful feedback. Lin was supported by Yale-NUS Startup Grant; part of the work was done when Lin was at Oxford supported by EPSRC (H026878). Zhou was supported by ARC (FT110100629).

\section{Preliminaries}

General Notations: We use log (resp. ln) to denote logarithm in base 2 (resp. natural logarithm). We use the standard interval notations to denote a subset of integers within that interval. For example, $[i, j)$ denotes the set $\{k \in \mathbb{Z}: i \leq k<$ $j\}$. Likewise, for each positive integer $n$, we use $[n]$ to denote the set $\{1, \ldots, n\}$. We shall also extend arithmetic operations to sets of numbers in the usual way: whenever $S_{1}, S_{2} \subseteq \mathbb{Z}$, we define $S_{1}+S_{2}:=\left\{s_{1}+s_{2}: s_{1} \in S_{1}, s_{2} \in S_{2}\right\}$ and $S_{1} S_{2}:=\left\{s_{1} \times s_{2}: s_{1} \in S_{1}, s_{2} \in S_{2}\right\}$. In the context of arithmetic over $2^{\mathbb{Z}}$, we will treat a number $n \in \mathbb{N}$ as the singleton set $\{n\}$. That way, for $a, b \in \mathbb{N}$, the notation $a+b \mathbb{Z}$ refers to the arithmetic progression $\{a+b c: c \in \mathbb{Z}\}$, where $a$ (resp. $b$ ) is called the offset (resp. period) of the arithmetic progression. Likewise, for a subset $S \subseteq \mathbb{N}$, we use $\operatorname{gcd}(S)$ to denote the greatest common divisor of $S$.

We will use standard notations from formal language theory. Let $\Gamma$ be an alphabet whose elements are called letters. A word (or a string) $w$ over $\Gamma$ is a finite sequence of elements from $\Gamma$. We use $\Gamma^{*}$ to denote the set of all words over $\Gamma$. The length of $w$ is denoted by $|w|$. Given a word $w=a_{1} \ldots a_{n}$, the notation $w[i, j]$ denotes the subword $a_{i} \ldots a_{j}$. For a sequence $\sigma=i_{1}, \ldots, i_{k} \in[n]^{*}$ of distinct indices of $w$, we write $w[\sigma]$ to denote the word $a_{i_{1}} \ldots a_{i_{k}}$. We also define $\operatorname{RS}(w)$ to be $a_{n} a_{1} a_{2} \ldots a_{n-1}$, i.e., the word $w$ cyclically right-shifted.

Number Theory: In the sequel, we will use some standard results in number theory and algorithmic number theory. The first result is Linear Congruence Theorem and its application to solving a system of linear congruences. The second result is Chinese Remainder Theorem.

Linear Congruence Theorem (e.g. see [13, Chapter 31.4] or [26, Theorem 4.5]) gives a fast method of determining whether an equation of the form $a x \equiv b$ $(\bmod n)$ is solvable and, whenever it is solvable, the set of solutions to $x$.

Lemma 1 (Linear Congruence Theorem) The equation $a x \equiv b(\bmod n)$ is solvable for the unknown $x$ iff $d \mid b$, where $d=\operatorname{gcd}(a, n)$. Furthermore, if it is solvable, then the set of solutions equals $x_{0}+(n / d) \mathbb{Z}$, for some $x_{0} \in[0, n / d)$ that can be computed in time $O(\log n)$ (assuming constant-time arithmetic operations).

An immediate application of Linear Congruence Theorem is to determine the set of solutions to a system of linear congruences. A system of linear congruence equations is a relation of the form $\bigwedge_{i=1}^{m} x \equiv a_{i}\left(\bmod b_{i}\right)$. [In general, a system of linear congruence equations might take an equation of the form $a x \equiv b(\bmod n)$, but we do not need this general form in the sequel.] The set of solutions $x \in \mathbb{Z}$ to this system is denoted by $\llbracket \bigwedge_{i=1}^{m} x \equiv a_{i}\left(\bmod b_{i}\right) \rrbracket$, which equals $\bigcap_{i=1}^{m}\left(a_{i}+b_{i} \mathbb{Z}\right)$. The system is solvable if the solution set is nonempty. We use FALSE to denote $x \equiv 0(\bmod 2) \wedge x \equiv 1(\bmod 2)$, which is not solvable. 
Proposition 1 For any solvable system of linear congruence equations $\varphi(x):=$ $\bigwedge_{i=1}^{m} x \equiv a_{i}\left(\bmod b_{i}\right)$, we have $\llbracket \varphi(x) \rrbracket=\llbracket x \equiv a(\bmod b) \rrbracket$ for some $a, b \in \mathbb{Z}$. Furthermore, there exists an algorithm which computes $a, b$ in linear time (assuming constant-time arithmetic operations).

Proposition 1 is witnessed by Algorithm 1, which is simply a repeated application of Linear Congruence Theorem.

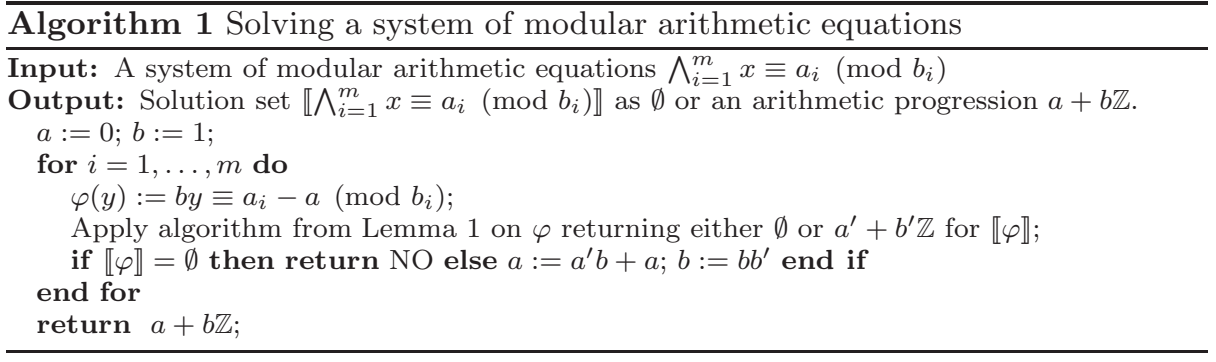

Remark 1 The number of bits that is used to maintain $a$ and $b$ in the worst case is linear in the size $\sum_{j=1}^{m}\left(\log a_{j}+\log b_{j}\right)$ of the input. This justifies treating a single arithmetic operation as a constant-time operation. We will discuss bit complexity in Section 4

In the sequel, we will also use Chinese Remainder Theorem (e.g. see [13, Section 31.5] or [26, Theorem 2.6]).

Proposition 2 (Chinese Remainder Theorem) Let $n_{1}, \ldots, n_{k}$ be pairwise relatively prime positive integers, and $n=\prod_{i=1}^{k} n_{i}$. The ring $\mathbb{Z}_{n}$ and the direct product of rings $\mathbb{Z}_{n_{1}} \times \cdots \times \mathbb{Z}_{n_{k}}$ are isomorphic under the function $\sigma: \mathbb{Z} \rightarrow$ $\mathbb{Z}_{n_{1}} \times \cdots \times \mathbb{Z}_{n_{k}}$ with $\sigma(x):=\left(x \bmod n_{1}, \ldots, x \bmod n_{k}\right)$ for each $x \in \mathbb{Z}$.

Groups: We briefly recall basic concepts from group theory and permutation groups (cf. see [8]). A group $G$ is a pair $(S, \cdot)$, where $S$ is a set and $\cdot:(S \times S) \rightarrow S$ is a binary operator satisfying: (i) associativity (i.e. $\left.g_{1} \cdot\left(g_{2} \cdot g_{3}\right)=\left(g_{1} \cdot g_{2}\right) \cdot g_{3}\right)$, (ii) the existence of a (unique) identity element $e \in S$ such that $g \cdot e=e \cdot g=g$ for all $g \in S$, and (iii) closure under inverse (i.e. for each $g \in G$, there exists $g^{-1} \in G$ such that $g \cdot g^{-1}=g^{-1} \cdot g=e$ ). When it is clear from the context, we will write $g \cdot g^{\prime}$ as $g g^{\prime}$. The order $\operatorname{ord}(G)$ of the group $G$ is defined to be the number $|S|$ of elements in $G$. This paper concerns only finite groups, i.e., groups $G$ with $\operatorname{ord}(G)=|S| \in \mathbb{N}$. For each $n \in \mathbb{N}$, we define $g^{n}$ by induction: (i) $g^{0}=e$, and (ii) $g^{n}=g^{n-1} \cdot g$. The order $\operatorname{ord}(g)$ of $g \in G$ is the least positive integer $n$ such that $g^{n}=e$.

A subgroup $H$ of $G=(S, \cdot)$ (denoted as $H \leq G$ ) is any group $\left(S^{\prime}, \cdot H\right.$ ) such that $S^{\prime} \subseteq S$ and $\cdot H$ and · agree on $S^{\prime}$. Lagrange's Theorem states that the order $\operatorname{ord}(H)$ of $\bar{H}$ divides the order $\operatorname{ord}(G)$ of $G$. Given any subset $X \subseteq S$, the subgroup $\langle X\rangle$ of $G$ generated by $X$ consists of those elements of $G$ which can be expressed as a finite product of elements of $X$ and their inverses. If $H=\langle X\rangle$, then $X$ is said to generate $H$. A cyclic group is a group generated by a singleton set $X=\{g\}$.

An action of a group $G=(S, \cdot)$ on a set $Y$ is a function $\times: S \times Y \rightarrow Y$ such that for all $g, h \in S$ and $y \in Y:(1)(g h) \times y=g \times(h \times y)$, and (2) $e \times y=y$. The stabiliser of $x$ by $G$ is the subgroup $\operatorname{Stab}_{G}(x):=\{g \in G: g \times x=x\}$ of $G$. If $G$ is understood, $\operatorname{Stab}(x)$ will be used to denote $\operatorname{Stab}_{G}(x)$. The $(G$-)orbit containing $y$, 
denoted $G y$, is the subset $\{g \times y: g \in G\}$ of $Y$. The action $\times$ partitions the set $Y$ into $G$-orbits. When the meaning is clear, we shall omit mention of the operator $\times$, e.g, condition (2) above becomes $e y=y$.

Permutation Groups. A permutation on $[n]$ is any bijection $\pi:[n] \rightarrow[n]$. The set of all permutations on $[n]$ forms the (nth) full symmetry group $\mathcal{S}_{n}$ under functional composition. We shall use the notation Id to denote the identity element of each $\mathcal{S}_{n}$. A word $w=a_{0} \ldots a_{k-1} \in[n]^{*}$ containing distinct elements of $[n]$ (i.e. $a_{i} \neq a_{j}$ if $i \neq j$ ) can be used to denote the permutation that maps $a_{i} \mapsto a_{i+1} \bmod k$ for each $i \in[0, k)$ and fixes other elements of $[n]$. In this case, $w$ is called a cycle (more precisely, $k$-cycle or transpositions in the case when $k=2$ ), which we will often write in the standard notation $\left(a_{0}, \ldots, a_{k-1}\right)$ so as to avoid confusion. Observe that $w$ and $\operatorname{RS}(w)$ represent the same cycle $c$. We will however fix a particular ordering to represent $c$ (e.g. the word provided as input to the orbit problem). For this reason, if $\mathbf{v} \in \Gamma^{n}$ for some alphabet $\Gamma$, the notation $\mathbf{v}[c]$ is welldefined (see General Notations above), which means projections of $\mathbf{v}$ onto elements with indices in $c$, e.g., if $\mathbf{v}=(1,1,1,0)$ and $c=(1,4,2)$, then $\mathbf{v}[c]=(1,0,1)$. Any permutation can be written as a composition of disjoint cycles 8]. Each subgroup $G=(S, \cdot)$ of $\mathcal{S}_{n}$ acts on the set $\Gamma^{n}$ (over any finite alphabet $\Gamma$ ) under the group action of permuting indices, i.e., for each $\pi \in S$ and $\mathbf{v}=\left(a_{1}, \ldots, a_{n}\right) \in \Gamma^{n}$, we define $\pi \mathbf{v}:=\left(a_{\pi^{-1}(1)}, \ldots, a_{\pi^{-1}(n)}\right)$.

Complexity Analysis: We will assume that permutations will be given in the input as a composition of disjoint cycles. It is easy to see that permutations can be converted back and forth in linear time from such representations and the representations of permutations as functions. The size $\|n\|$ of a number $n \in \mathbb{N}$ is defined to be the length of the binary representation of $n$, which is $\lfloor\log n\rfloor+1$. The size $\|c\|$ of a cycle $c=\left(a_{1}, \ldots, a_{k}\right)$ on $[n]$ is defined to be $\sum_{i=1}^{k}\left\|a_{i}\right\|$ (in contrast, the length $|c|$ of $c$ is $k$ ). For a permutation $\pi=c_{1} \cdots c_{m}$ where each $c_{i}$ is a cycle, the size $\|\pi\|$ of $\pi$ is defined to be $\sum_{i=1}^{m}\left\|c_{i}\right\|$. We will use standard asymptotic notations from analysis of algorithms (big-O and little-o), cf. [13. We also use the standard $\sim$ notation: $f(n) \sim g(n)$ iff $\lim _{n \rightarrow \infty} f(n) / g(n)=1$. We will use the standard RAM model that is commonly used when analysing the complexity of algorithms (cf. [13]). In Section 3, we will assume that integer arithmetic takes constant time. Later in Section 4. we will use the bit complexity model (cf. [13]), wherein the running time is measured in the number of bit operations.

\section{Reducing to solving a system of linear congruence equations}

The main result of the paper is:

Theorem 1 There is a linear-time algorithm for solving the orbit problem when the acting group is cyclic.

In this section, we will prove this theorem assuming constant-time arithmetic operations. In the next section, we will show that this theorem still holds for the bit complexity model.

Before we proceed to the algorithm, the following proposition shows why the naive algorithm that checks whether $g^{i} \mathbf{v}=\mathbf{w}$, for a given permutation $g \in \mathcal{S}_{n}$ and for each $i \in[0, \operatorname{ord}(g))$, actually runs in exponential time. 
Proposition 3 There exists a sequence $\left\{G_{i}\right\}_{i=1}^{\infty}$ of cyclic groups $G_{i}=\left\langle g_{i}\right\rangle$ such that $\operatorname{ord}\left(g_{i}\right)$ is exponential in the size $\left\|g_{i}\right\|$ of the permutation $g_{i}$.

Proof Let $p_{n}$ denote the $n$th prime. The Prime Number Theorem states that $p_{n} \sim$ $n \log n$ (cf. [19]). For each $i \in \mathbb{Z}_{>0}$, we define a cycle $c_{i}$ of length $p_{i}$ by induction on $i$. For $i=1$, let $c_{1}=(1,2)$. Suppose that $c_{i-1}=(j, \ldots, k)$. In this case, we define $c_{i}$ to be the cycle $\left(k+1, \ldots, k+p_{i}\right)$. To define the sequence $\left\{g_{i}\right\}_{i=1}^{\infty}$ of permutations, simply let $g_{i}=\Pi_{j=1}^{i} c_{i}$. For example, we have $g_{3}=(1,2)(3,4,5)(6,7,8,9,10)$. Since $c_{i}$ 's are disjoint, the order ord $\left(g_{i}\right)$ of $g_{i}$ is the smallest positive integer $k$ such that $c_{j}^{k}=$ Id for all $j \in[i]$. If $S_{j}$ denotes the set of integers $k$ satisfying $c_{j}^{k}=\mathrm{Id}$, then $\operatorname{ord}\left(g_{i}\right)$ is precisely the smallest positive integer in the set $\bigcap_{j=1}^{i} S_{j}$. It is easy to see that $S_{j}=p_{j} \mathbb{Z}$, which is the set of solutions to the linear congruence equation $x \equiv 0\left(\bmod p_{j}\right)$. Therefore, by the Chinese Remainder Theorem (cf. Propositon 2), the set $\bigcap_{j=1}^{i} S_{j}$ coincides with the arithmetic progression $t_{i} \mathbb{Z}$ with $t_{i}:=\prod_{j=1}^{i} p_{j}$. This implies that ord $\left(g_{i}\right)=t_{i}$. Now the number $t_{i}$ is also known as the ith primorial number [1] with $t_{i} \sim e^{(1+o(1)) i \log i}$, which is a corollary of the Prime Number Theorem. On the other hand, the size of $g_{i}$ is $\sum(i):=\sum_{j=1}^{i} p_{i}$, which is known to be $\sim \frac{1}{2} i^{2} \ln i$ (cf. [4]). Therefore, $\operatorname{ord}\left(g_{i}\right)$ is exponential in $\left\|g_{i}\right\|$ as desired.

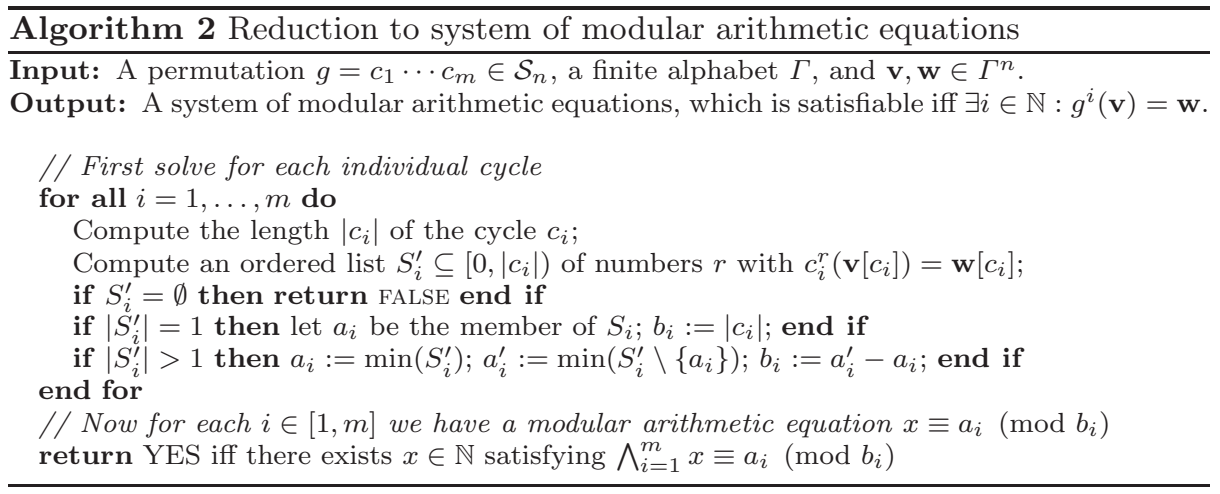

Our linear-time reduction that witnesses Theorem 1 is given in Algorithm 2 , In this algorithm, the acting group is $G=\langle g\rangle$ with $g \in \mathcal{S}_{n}$, expressed as a composition of disjoint cycles in a standard way, say, $g=c_{1} c_{2} \cdots c_{m}$ where each $c_{i}$ is a cycle. Also part of the input is two strings $\mathbf{v}=v_{1} \ldots v_{n}, \mathbf{w}=w_{1} \ldots w_{n} \in \Gamma^{n}$ over a finite alphabet $\Gamma$. The orbit problem is to check whether $f \mathbf{v}=\mathbf{w}$ for some $f \in G$, i.e., $f=g^{r}$ for some $r \in \mathbb{Z}$. Since $c_{i}$ 's are pairwise disjoint cycles, the question reduces to checking if there exists $r \in \mathbb{N}$ such that

$$
\forall i \in[1, m]:\left(c_{i}^{r} \mathbf{v}\right)\left[c_{i}\right]=\mathbf{w}\left[c_{i}\right]
$$

In other words, for each $i \in[1, m]$, applying the action $c_{i}^{r}$ to $\mathbf{v}$ gives us $\mathbf{w}$ when restricted to the indices in $c_{i}$. To simplify notations in the above equation, we fix a letter $a \in \Gamma$ and, for each $i \in[1, m]$, let the notation $\mathbf{v}_{i}\left(\operatorname{resp} . \mathbf{w}_{i}\right)$ denote the string $\mathbf{v}$ (resp. w) in which all letters but those in positions $c_{i}$ are replaced by $a$. The equation above, therefore, amounts to

$$
\forall i \in[1, m]: c_{i}^{r} \mathbf{v}_{i}=\mathbf{w}_{i}
$$


Essentially, Algorithm 2 sequentially goes through each cycle $c_{i}$ and computes the set $S_{i}$ of solutions $r$ to $c_{i}^{r} \mathbf{v}_{i}=\mathbf{w}_{i}$ as the set of solutions to the linear congruence equation $x \equiv a_{i}\left(\bmod b_{i}\right)$. Therefore, the set of solutions to $\left(^{*}\right)$ is precisely the set of solutions to the system of congruence equations $\bigwedge_{i=1}^{m} x \equiv a_{i}\left(\bmod b_{i}\right)$, which by Proposition 1 can be solved in linear time.

To compute $S_{i}$, we first prove a simple canonical form for $S_{i}$.

Lemma 2 For each $i=1, \ldots, m$, either $S_{i}=\emptyset$ or $S_{i}=a_{i}+b_{i} \mathbb{Z}$ for some $a_{i} \in\left[0, b_{i}\right)$ and $b_{i} \in\left(0,\left|c_{i}\right|\right]$ where $b_{i}$ divides $\left|c_{i}\right|$.

Proof Let $G_{i}=\left\langle c_{i}\right\rangle$ be the group generated by $c_{i}$. Consider the stabiliser $H:=$ $\operatorname{Stab}\left(\mathbf{v}_{i}\right)$ of $\mathbf{v}_{i}$ by $G_{i}$. Then, $H$ is a subgroup of $G_{i}$. Since $G_{i}$ is a cyclic group of order $\left|c_{i}\right|, H$ is a cyclic group generated by some element $h=c_{i}^{k}$, where $k$ is the smallest integer in $\left(0,\left|c_{i}\right|\right]$ such that $c_{i}^{k} \in H$. It is known that $k$ must be a divisor of $\left|c_{i}\right|$. This implies that the orbit containing $\mathbf{v}_{i}$ consists of precisely $k$ elements $\mathbf{v}_{i}, c_{i} \mathbf{v}_{i}, \ldots, c_{i}^{k-1} \mathbf{v}_{i}$.

Suppose that $S_{i} \neq \emptyset$. Let $s$ be the smallest nonnegative integer in $S_{i}$, i.e., $c_{i}^{s} \mathbf{v}_{i}=\mathbf{w}_{i}$. Then, $s \in[0, k)$. We claim that $S_{i}=s+k \mathbb{Z}$. We have $s+k \mathbb{Z} \subseteq S_{i}$ since $c_{i}^{s+k n} \mathbf{v}_{i}=c_{i}^{s}\left(c_{i}^{k n} \mathbf{v}_{i}\right)=c_{i}^{s} \mathbf{v}_{i}$. Conversely, if $t \in S_{i}$, then $c^{t} \mathbf{v}_{i}=c^{p} \mathbf{v}_{i}$, where $p$ is the smallest nonnegative integer such that $p \equiv t(\bmod k)$. Then, it must be the case that $p=s$ since $\mathbf{v}_{i}, c_{i} \mathbf{v}_{i}, \ldots, c_{i}^{k-1} \mathbf{v}_{i}$ are all different. Thus, it follows that $S_{i} \subseteq s+k \mathbb{Z}$. Letting $a_{i}=s$ and $b_{i}=k$ completes the proof.

In view of Lemma 2 it suffices to show how to determine if $S_{i} \neq \emptyset$ and, if so, compute $a_{i}$ and $b_{i}$ in time $O\left(\left\|c_{i}\right\|\right)$. We first compute the length $\left|c_{i}\right|$ of the cycle $c_{i}$, which can be done in time $O\left(\left\|c_{i}\right\|\right)$. [This is the same as how to compute the length of a list.] We proceed by computing representatives $S_{i}^{\prime} \subseteq\left[0,\left|c_{i}\right|\right)$ for $S_{i}$. This suffices to compute $a_{i}$ and $b_{i}$ since:

- If $S_{i}^{\prime}=\emptyset$, then $S_{i}=\emptyset$.

- If $S_{i}^{\prime}=\{a\}$, then $S_{i}=a+\left|c_{i}\right| \mathbb{Z}$.

- If $\left|S_{i}^{\prime}\right|>1$, then $S_{i}=a_{i}+b_{i} \mathbb{Z}$, whenever $a_{i}$ and $a_{i}+b_{i}$ are the two smallest numbers in $S_{i}^{\prime}$.

This case-by-case treatment is reflected in Line 3 -Line 5 within the for-loop in Algorithm 2. To compute $S_{i}^{\prime}$, we collect a subset of numbers $h \in\left[0,\left|c_{i}\right|\right)$ such that $c_{i}^{h} \mathbf{v}_{i}=\mathbf{w}_{i}$. A quadratic algorithm for this is easy to come up with: sequentially go through $h \in\left[0,\left|c_{i}\right|\right)$ while computing the current $c_{i}^{h}$, and save $h$ if $c_{i}^{h} \mathbf{v}_{i}=\mathbf{w}_{i}$ holds. One way to obtain a linear-time algorithm is to reduce our problem to the string searching problem: given a "text" $T \in \Sigma^{*}$ (over some finite alphabet $\Sigma$ ) and a "pattern" $P \in \Sigma^{*}$, find all positions $i$ in $T$ such that $T[i, i+|P|]=P$. This problem is solvable in time $O(|T|+|P|)$ by Knuth-Morris-Pratt (KMP) algorithm (e.g. see 13]).

We now show how to reduce our problem to the string searching problem in linear time. We will also use the following running example to illustrate the reduction: $c=(6,5,7,3,2,1), \mathbf{v}=\underline{010001111}$, and $\mathbf{w}=\underline{101110001}$, where the positions in $\mathbf{v}$ and $\mathbf{w}$ that are modified by $c$ are underlined. Below, we will work with the equivalent equation $\left(c_{i}^{r} \mathbf{v}\right)\left[c_{i}\right]=\mathbf{w}\left[c_{i}\right]$ (i.e. instead of $c_{i}^{r} \mathbf{v}_{i}=\mathbf{w}_{i}$ ). Suppose that $c:=c_{i}=\left(j_{1}, \ldots, j_{k}\right)$. We have $\mathbf{v}[c]=v_{j_{1}} \ldots v_{j_{k}}$ and $\mathbf{w}[c]=w_{j_{1}} \ldots w_{j_{k}}$.

Lemma $3(c \boldsymbol{v})[c]=\operatorname{RS}(\boldsymbol{v}[c])$. 
In other words, if $\operatorname{Dom}(c)=\left\{j_{1}, \ldots, j_{k}\right\}$, the effect of $c$ on $\mathbf{v}$ when restricted to $\operatorname{Dom}(c)$ coincides with applying a cyclical right shift on the string $\mathbf{v}[c]$. Following our running example, it is easy to check that $\mathbf{v}[c]=101010$ and $(c \mathbf{v})[c]=$ $\operatorname{RS}(\mathbf{v}[c])=010101$.

Proof (of Lemma 3) Let $\mathbf{u}=u_{1} \ldots u_{k}:=(c \mathbf{v})[c]$ and $\mathbf{u}=u_{1}^{\prime} \ldots u_{k}^{\prime}:=\operatorname{RS}(\mathbf{v}[c])$. It suffices to show that $u_{t}=u_{t}^{\prime}$ for all $t \in \mathbb{Z}_{k}$. By definition of RS, it follows that $u_{t}^{\prime}=v_{j_{t-1}}$. Now suppose that $\mathbf{v}^{\prime}=v_{1}^{\prime} \ldots v_{n}^{\prime}:=c \mathbf{v}$. Then

$$
v_{j}^{\prime}:=\left\{\begin{array}{cc}
v_{j} & \text { if } j \notin \operatorname{Dom}(c) \\
v_{j^{\prime}} & \text { if } j \in \operatorname{Dom}(c) \text { and, for some } t \in \mathbb{Z}_{k}, j=j_{t+1} \text { and } j^{\prime}=j_{t} .
\end{array}\right.
$$

So, for $t \in \operatorname{Dom}(c)$, we have $u_{t}=((c \mathbf{v})[c])[t]=\left(\mathbf{v}^{\prime}[c]\right)[t]=v_{j_{t}}^{\prime}=v_{j_{t-1}}$. This proves that $u_{t}=u_{t}^{\prime}$

Lemma 4 For each $r \in \mathbb{N}$, we have $\left(c^{r} \boldsymbol{v}\right)[c]=\operatorname{RS}^{r}(\boldsymbol{v}[c])$.

Proof This lemma can be proved by induction on $r \in \mathbb{N}$. The base case $r=0$ is vacuous. For the induction case, we assume the induction hypothesis: $c^{r-1} \mathbf{v}=$ $\operatorname{RS}^{r-1}(\mathbf{v}[c])$. It follows that

$$
\left(c^{r} \mathbf{v}\right)[c]=\left(c\left(c^{r-1} \mathbf{v}\right)\right)[c]=\operatorname{RS}\left(\left(c^{r-1} \mathbf{v}\right)[c]\right)=\operatorname{RS}\left(\operatorname{RS}^{r-1}(\mathbf{v}[c])\right)=\operatorname{RS}^{r}(\mathbf{v}[c]) .
$$

The third equality is by Lemma 3, while the fourth equality is by the induction hypothesis. This completes the proof.

Define the text $T:=\mathbf{v}[c] \mathbf{v}[c]$ and the pattern $P:=\mathbf{w}[c]$. Observe that, for each $r \in[k], P$ is matched at position $r$ in $T$ iff $\operatorname{RS}^{r-1}(\mathbf{v}[c])=\mathbf{w}[c]$. Therefore, after running the KMP algorithm with the solution set $S^{\prime}$, the set $S_{i}^{\prime}$ will be $\left\{r-1: r \in S^{\prime}\right\}$. Observe that this step takes time $O(|T|+|P|)=O(\|c\|)$.

Example 2 Continuing with our running example, it follows that $T=\mathbf{v}[c] \mathbf{v}[c]=$ 101010101010 and $P=\mathbf{w}[c]=010101$. We see that $P$ matches $T$ at positions $S^{\prime}=\{2,4,6\}$. This implies that $S_{i}^{\prime}=\{1,3,5\}$ and so the set $S_{i}$ of solutions $r \in \mathbb{Z}$ to the equation $c^{r} \mathbf{v}_{i}=\mathbf{w}_{i}$ is $1+2 \mathbb{Z}$.

Summing up. To sum up, the time spent computing the linear congruence equation $x \equiv a_{i}\left(\bmod b_{i}\right)$ for each $i \in[1, m]$ is $O\left(\left\|c_{i}\right\|\right)$. Therefore, our reduction runs in time $O\left(\sum_{i=1}^{m}\left\|c_{i}\right\|\right)=O(\|g\|)$, which is linear in input size. Therefore, invoking Proposition 1 on the resulting system of linear congruence equations, we obtain the set of solutions to $(*)$ in linear time.

Example 3 Let us continue with our running example. Let

$$
g_{1}:=c(4,8)=(6,5,7,3,2,1)(4,8), \quad g_{2}:=c(4,8,9)=(6,5,7,3,2,1)(4,8,9)
$$

Then, running Algorithm2 2 on $g_{1}$ yields the system $x \equiv 1(\bmod 2) \wedge x \equiv 1(\bmod 2)$, which is equivalent to $x \equiv 1(\bmod 2)$. Running Algorithm 2 on $g_{2}$ yields the system $x \equiv 1(\bmod 2) \wedge x \equiv 1(\bmod 3)$. Both systems are solvable. 


\section{Making do with linearly many bit operations}

Thus far, we have assumed that arithmetic operations take constant time. In this section, since Algorithm 1 makes a substantial use of basic arithmetic operations, we will revisit this assumption. It turns out that, although our reduction (Algorithm 21) to solving a system of linear congruence equations runs in linear time in the bit complexity model, the algorithm for solving the system of equations (Algorithm 1) uses at least a cubic number of bit-arithmetic operations. The main results in this section are two-fold: (1) on inputs given by our reduction, Algorithm 1 runs in sublinear time (more precisely, $O\left(\log ^{5} n\right)$ ) on average in the bit complexity model, and (2) there exists another algorithm for solving a system of linear congruence equations (with numbers in the input represented in unary) that runs in linear time in the bit complexity model in the worst case.

We begin with two lemmas that provide the running time of Algorithm 2 and Algorithm 1 in the bit complexity model.

Lemma 5 Algorithm 2 runs in linear time in the bit complexity model.

Proof On $i$ th iteration, the number $\left|c_{i}\right|$ is stored in binary counter and can be computed by counting upwards from 0 and incrementing by 1 as we go through the elements in $c_{i}$. Although a single increment by 1 might take $O\left(\left|c_{i}\right|\right)$ bit operations in the worst case (since we have to propagate the carry bit), it is known (e.g. see [13. Chapter 17, p. 454]) that the entire sequence of operations actually takes time $O\left(\left|c_{i}\right|\right)$. Finally, since addition and substraction of two numbers can easily be performed in $O(\beta)$ time on numbers that use at most $\beta$ bits, the operation $b_{i}:=$ $a_{i}^{\prime}-a_{i}$ on the last line of the iteration takes at most $O\left(\log \left|c_{i}\right|\right)$ time. Therefore, accounting for all the cycles, the algorithm takes $\sum_{i=1}^{m} O\left(\left\|c_{i}\right\|\right)=O\left(\sum_{i=1}^{m}\left\|c_{i}\right\|\right)=$ $O(\|g\|)$, which is linear in the input size.

Lemma 6 On an input $\bigwedge_{i=1}^{m} x \equiv a_{i}\left(\bmod b_{i}\right)$ with $N=\max \left\{b_{i}: i \in[1, m]\right\}$, Algorithm 1 uses at most $m \log N$ bits to store any numeric variables. Furthermore, the algorithm runs in time $O\left(m^{3} \log ^{2} N\right)$ in the bit complexity model.

Proof On $i$ th iteration, the number of bits used to store $a$ and $b$ grow by at most $\log b_{i}$. On the other hand, the invariant that $a^{\prime}, b^{\prime} \in\left[0, b_{i}\right)$ is always maintained on the $i$ th iteration and so they only need at most $\log N$ bits to represent throughout the algorithm. Hence, the algorithm uses $M=O(m \log N)$ bits to store $a, b, a^{\prime}$, and $b^{\prime}$. Extended Euclidean Algorithm runs in time $O\left(M^{2}\right)$ on inputs where each number uses at most $M$ bits (cf. [13, Problem 31-2]), which also bounds the time it takes on each iteration. Therefore, the algorithm takes at most $O\left(m M^{2}\right)=$ $O\left(m^{3} \log ^{2} N\right)$ in the bit complexity model.

We now provide an average case analysis of the running time of Algorithm 1 on system of linear congruence equations given by our reduction. The input to the orbit problem over cyclic groups includes a permutation $g \in S_{n}$ and two vectors $\mathbf{v}, \mathbf{w} \in \Gamma^{n}$. We briefly recall the setting of average-case analysis (cf. [25]). Let $\Pi_{N}$ be the set of all inputs to the algorithm of size $N$. Likewise, let $\Sigma_{N}$ be the sum of the costs (i.e. running time) of the algorithm on all inputs of size $N$. Hence, if $\Pi_{N, k}$ is the cost of the algorithm on input of size $N$ with running time $k$, then $\Sigma_{N}=\sum_{k} k \Pi_{N, k}$. The average case complexity of the algorithm is defined to be $\Sigma_{N} / \Pi_{N}$. 
Theorem 2 The expected running time of Algorithm 1 in the bit complexity model on inputs provided by Algorithm 2 is $O\left(\log ^{5} n\right)$.

Proof The size of a single permutation $g \in S_{n}$ is $O(n)$ and additionally $\Pi_{n}=$ $\left|S_{n}\right|=n$ !. Suppose that $g$ has $k$ cycles (say, $g=c_{1} \cdots c_{k}$ ). Then, Algorithm 2 produces a system of equations $\bigwedge_{i=1}^{k} x \equiv a_{i}\left(\bmod b_{i}\right)$, where $a_{i}, b_{i} \in\left[0,\left|c_{i}\right|\right)$. By Lemma 6] Algorithm 1 takes $O\left(k^{3} \log ^{2} n\right)$ time in the bit complexity model, since $N:=\max \left\{b_{i}: i \in[1, m]\right\} \leq n$. In addition, the number of permutations in $S_{n}$ with $k$ cycles is precisely the definition of the unsigned Stirling number of the first kind $\left[\begin{array}{l}n \\ k\end{array}\right]$. Therefore, we have $\Sigma_{n}=O\left(\sum_{k=1}^{n}\left(k^{3} \log ^{2} n\right)\left[\begin{array}{l}n \\ k\end{array}\right]\right)=$ $O\left(\log ^{2} n \sum_{k=1}^{n} k^{3}\left[\begin{array}{l}n \\ k\end{array}\right]\right)$. Therefore, it suffices to show that $\frac{1}{n !} \sum_{k=1}^{n} k^{3}\left[\begin{array}{l}n \\ k\end{array}\right] \sim$ $c \log ^{3} n$ for a constant $c$. The proof can be found in the appendix.

Finally, we will now give our final main result of this section.

Theorem 3 There exists a linear-time algorithm in the bit complexity model for solving a system of linear congruence equations when the input numbers are represented in unary.

We now provide an algorithm that witnesses the above theorem. Let $\bigwedge_{i=1}^{m} x \equiv a_{i}$ $\left(\bmod b_{i}\right)$ be the given system of equations. With unary representation of numbers, the size $N_{i}$ of the equation $x \equiv a_{i}\left(\bmod b_{i}\right)$ is $a_{i}+b_{i}$. We use $n$ to denote the total number of bits in the system of equations. Initially, we compute a binary representation of all the numbers $a_{i}$ 's, $b_{i}$ 's, and $n$ as in the proof of Lemma 5 . which takes linear time. Next we factorise all the numbers $b_{i}$ into a product of distinct prime powers $p_{j_{i 1}}^{e_{i 1}} \cdots p_{j_{i t_{i}}}^{e_{i t_{i}}}$, where $p_{j}$ stands for the $j$ th prime and all $e_{i j}$ 's are positive integers. This can be done in time $O\left(\sqrt{N_{i}} \log ^{2} N_{i}\right)$. To obtain this time bound, we can use any unconditiona 3 deterministic factorisation methods like Strassen's algorithm, whose complexity was shown in 6] (cf. also see [14) to be $O\left(f\left(N^{1 / 4} \log N\right)\right)$ for factoring a number $N$, where $f(M)$ is the number of bit operations required to multiply two numbers with $M$ bits. The standard (highschool) multiplication algorithm runs in quadratic time giving us $f(M)=O\left(M^{2}\right)$, which suffices for our purposes. This shows that Strassen's algorithm runs in time $O\left(N^{1 / 2} \log ^{2} N\right)$. [In practice, do factoring using the general number field sieve (cf. [13]), which performs extremely well in practice, though its complexity requires some unproven number-theoretic assumptions.]

Next, following Chinese Remainder Theorem (CRT), compute $z_{i j}:=a_{i} \bmod p_{i j}^{e_{i j}}$ for each $j \in\left[1, t_{i}\right]$. Let us analyse the time complexity for performing this. Each $z_{i j}$ can be computed by a standard algorithm (e.g. see [13]) in time quadratic in the number of bits used to represent $a_{i}$ and $p_{i j}^{e_{i j}}$. Since each of these numbers use at most $\log N_{i}$ bits, each $z_{i}$ can be computed in time $O\left(\log ^{2} N_{i}\right)$, which is $o\left(N_{i}\right)$. In addition, since $e_{i j}>1$ for each $j \in\left[1, t_{i}\right]$, it follows that $t_{i}=O\left(\log N_{i}\right)$. This means that the total time it takes to compute $\left\{z_{i j}: j \in\left[1, t_{i}\right]\right\}$ is $O\left(\log ^{3} N_{i}\right)$, which is also $o\left(N_{i}\right)$. So, computing this for all $i \in[1, m]$ takes time $O\left(\sum_{i=1}^{m} \log ^{3} N_{i}\right)$, which is at most linear in the input size.

\footnotetext{
3 This means that the bound does not depend on any number-theoretic assumptions.
} 
In summary, for each $i \in[1, m]$, we obtained the following system of equations, which is equivalent to $x \equiv a_{i}\left(\bmod b_{i}\right)$ by CRT:

$$
x \equiv z_{i 1} \quad\left(\bmod p_{i 1}^{e_{i 1}}\right) \wedge \cdots \cdots \wedge \quad x \equiv z_{i t_{i}}\left(\bmod p_{i t_{i}}^{e_{i t_{i}}}\right)
$$

The final step is to determine if there exists a number $x \in \mathbb{N}$ that satisfies each $\left(E_{i}\right)$, for all $i \in[1, m]$. Loosely, we will go through all the equations and make sure that there is no conflict between any two equations whose periods are powers of the same prime number, i.e., $x \equiv a(\bmod b)$ and $x \equiv a^{\prime}\left(\bmod b^{\prime}\right)$ such that $b=p^{i}$ and $b^{\prime}=p^{i^{\prime}}$ for some prime $p$ and $i, i^{\prime} \in \mathbb{Z}_{>0}$. In order to achieve this in linear-time in the bit complexity model, one has to store these equations in the memory (in the form of lookup tables) and carefully perform the lookup operations while looking for a conflict. To this end, we first compute $p_{\max }=\max \left\{p_{i j}: i \in[1, m], j \in\left[1, t_{i}\right]\right\}$ and $e_{\max }=\max \left\{e_{i j}: i \in[1, m], j \in\left[1, t_{j}\right]\right\}$.

Lemma $7 p_{\max }$ and $e_{\max }$ can be computed using $O(n)$ many bit operations.

Proof The algorithm for computing $p_{\max }$ and $e_{\max }$ is a slight modification of the standard algorithm that computes the maximum number in a list, which sequentially goes through the list $n_{1}, \ldots, n_{m}$ while keeping the maximum number $n_{\max }$ in the sublist explored so far. To ensure linear-time complexity, we have to make sure that when comparing the values of $n_{i}$ and $n_{\max }$, we explore at most $n_{i}$ bits of $n_{\max }$ (since $n_{\max }$ is possibly much larger than $n_{i}$ ). This is easily achievable by assuming binary representation of these numbers without redundant leading 0s, e.g., the number 5 will be represented as 101, not 0101 or 00000101 . That way, we will only need to inspect $\log \left(n_{i}\right)$ bits from $n_{\max }$ on the $i$ th iteration, which will give a total running time of $O\left(\sum_{i=1}^{m} \log \left(n_{i}\right)\right)$, which is linear in input size.

Next, keep one 1-dimensional array $A$ and one 2-dimensional array $B$ :

$$
A\left[1, \ldots, p_{\max }\right] \quad B\left[1, \ldots, p_{\max }\right]\left[1, \ldots, e_{\max }\right] .
$$

$A[k]$ and $B[k][e]$ will not be defined when $k$ is not a prime number. We will use $A[k]$ as a flag indicating whether some equation of the form $x \equiv z\left(\bmod k^{e}\right)$ has been visited, in which case $A[k]$ will contain $(z, e)$. In this case, we will use $B[k]\left[e^{\prime}\right]$ (with $e^{\prime} \leq e$ ) to store the value of $z \bmod k^{e^{\prime}}$.

We now elaborate how $A$ and $B$ are used when iterating over the equations in the system. Sequentially go through each system $\left(E_{i}\right)$ of equations. For each $i \in[1, m]$, sequentially go through each equation $x \equiv z_{i j}\left(\bmod p_{i j}^{e_{i j}}\right)$, for each $j \in\left[1, t_{i}\right]$, and check if $A\left[p_{i j}\right]$ is defined. If it is not defined, set $A\left[p_{i j}\right]:=\left(z_{i j}, e_{i j}\right)$ and compute $B\left[p_{i j}\right][l]=z_{i j} \bmod p^{l}$ for each $l \in\left[1, e_{i j}\right]$. If it is defined (say, $\left.A\left[p_{i j}\right]=(z, e)\right)$, then we analyse the constraints $x \equiv z\left(\bmod p_{i j}^{e}\right)$ and $x \equiv z_{i j}$ $\left(\bmod p_{i j}^{e_{i j}}\right)$ simultaneously. We compare $e$ and $e_{i j}$ resulting in three cases:

Case 1. $e=e_{i j}$. In this case, make sure that $z=z_{i j}$ otherwise the two equations (and, hence, the entire system) cannot be satisfied simultaneously.

Case $2 . e<e_{i j}$. In this case, make sure that $z_{i j} \equiv z\left(\bmod p_{i j}^{e}\right)$ (otherwise, unsatisfiable) and assign $A\left[p_{i j}\right]:=\left(z_{i j}, e_{i j}\right)$. For each $l \in\left[1, e_{i j}\right]$, update $B\left[p_{i j}\right][l]:=z_{i j} \bmod p_{i j}^{l}$.

Case 3. $e>e_{i j}$. In this case, make sure that $z_{i j} \equiv z\left(\bmod p_{i j}^{e_{i j}}\right)$ (otherwise, unsatisfiable). 
We now analyse the running time of this final step (i.e. when scanning through the subsystem $\left.\left(E_{i}\right)\right)$. To this end, we measure the time it takes to process each equation $x \equiv z_{i j}\left(\bmod p_{i j}^{e_{i j}}\right)$. There are two cases, which we will analyse in turn.

(Case I): when $A\left[p_{i j}\right]$ is not defined. In this case, setting $A\left[p_{i j}\right]$ takes constant time, while setting $B\left[p_{i j}\right][l]$ for all $l \in\left[1, e_{i j}\right]$ takes $O\left(e_{i j} \times\left(\log z_{i j}+\log p_{i j}^{e_{i j}}\right)^{2}\right)$ since computing $a \bmod b$ can be done in time quadratic in $\log (a)+\log (b)$. Since $e_{i j} \leq \log N_{i}$ and $z_{i j}, p_{i j} \leq N_{i}$, this expression can be simplified to $O\left(\log N_{i} \times\right.$ $\left.\log ^{2}\left(z_{i j} N_{i} p_{i j}\right)\right)=O\left(\log ^{3} N_{i}\right)$.

(Case II): when $A\left[p_{i j}\right]$ is already defined, e.g., $A\left[p_{i j}\right]=(z, e)$. In this case, we will compare the values of $e$ and $e_{i j}$. To ensure linear-time complexity, we will make sure that at most $\log \left(e_{i j}\right)$ bits from $e$ are read by using the trick from the proof of Lemma 7. For Case 1, we will need extra $O\left(\log z_{i j}\right)=O\left(\log N_{i}\right)$ time steps. For Case 2, we have $0 \leq z \leq p^{e_{i j}}$ and computing $z_{i j} \bmod p_{i j}^{e}$ can be done in time $O\left(\log ^{2} N_{i}\right)$ as before. Updating $B\left[p_{i j}\right][l]$ for all $l \in\left[1, e_{i j}\right]$ takes $O\left(\log ^{3} N_{i}\right)$ as in the previous paragraph. For Case 3 , since $e>e_{i j}$, we may access the value of $z \bmod p_{i j}^{e_{i j}}$ from $B\left[p_{i j}\right]\left[e_{i j}\right]$ in constant time and compare this with the value of $z_{i j}$. Since $z \in\left[0, p_{i j}^{e_{i j}}\right)$, this takes time $O\left(\log N_{i}\right)$.

In summary, either case takes time at most $O\left(\log ^{3} N_{i}\right)$. Therefore, accounting for the entire subsystem $\left(E_{i}\right)$, the algorithm incurs $O\left(\sum_{j=1}^{t_{i}} \log ^{3} N_{i}\right)=O\left(\log ^{4} N_{i}\right)$ time steps. Hence, accounting for all of the subsystems $E_{i}(i \in[1, m])$ the algorithm takes time $O\left(\sum_{i=1}^{m} \log ^{4} N_{i}\right)$, which is linear in the size of the input. This completes the proof of Theorem 3

Remark 2 The purpose of the 2-dimensional array $B$ above is to avoid superlinear time complexity for Case 3 . We can imagine a system of linear equations $\bigwedge_{i=1}^{m} x \equiv$ $a_{i}\left(\bmod b_{i}\right)$, where $a_{1}$ and $b_{1}$ are substantially larger than the other $a_{i}$ 's and $b_{i}$ 's $(i \in[2, m])$. In this case, without the lookup table $B$, checking whether $a_{i} \equiv a_{1}$ $\left(\bmod b_{i}\right)$ in Case 3 will require the algorithm to inspect the entire value of $a_{1}$, which prevents us from bounding the time complexity in terms of $a_{i}$ and will yield a superlinear time complexity for our algorithm.

\section{Future work}

Since an algorithm for the orbit problem will be invoked many times during an explicit-state model checking (in the worst case once each time a new state in the transition system is visited; cf. [27]), we believe that it is important to further identify efficiently solvable (preferably, in linear-time) subcases of the orbit problem. As mentioned in the Introduction, there are known classes of permutations groups whose orbit problem is polynomial-time solvable (e.g. $\Gamma_{d}$ which contains solvable groups). We propose the question of further identifying other classes of permutations groups whose orbit problem is solvable in linear time.

\section{References}

1. http://oeis.org/A002110 Primorial Numbers (The On-Line Encyclopedia of Integer Sequences)

2. Babai, L., Luks, E.M.: Canonical labeling of graphs. In: STOC, pp. 171-183 (1983) 
3. Babai, L., Moran, S.: Arthur-merlin games: A randomized proof system, and a hierarchy of complexity classes. J. Comput. Syst. Sci. 36(2), 254-276 (1988). DOI 10.1016/ 0022-0000(88)90028-1. URL http://dx.doi.org/10.1016/0022-0000(88)90028-1

4. Bach, E., Shallit, J.: Algorithmic Number Theory, Foundations of Computing, vol. 1. The MIT Press (1996)

5. Benjamin, A.T., Preston, G.O., Quinn, J.J.: A stirling encounter with harmonic numbers. Mathematics Magazine 75, 95-103 (2002)

6. Bostan, A., Gaudry, P., Schost, É.: Linear Recurrences with Polynomial Coefficients and Application to Integer Factorization and Cartier-Manin Operator. SIAM J. Comput. 36(6), 1777-1806 (2007)

7. Brualdi, R.A.: Combinatorial matrix classes. Encyclopedia of Mathematics and Its Applications 108. Cambridge University Press (2006)

8. Cameron, P.J.: Permutation Groups. London Mathematical Society Student Texts. Cambridge University Press (1999)

9. Clarke, E.M.: The birth of model checking. In: 25 Years of Model Checking (2008)

10. Clarke, E.M., Emerson, E.A., Jha, S., Sistla, A.P.: Symmetry reductions in model checking. In: CAV, pp. 147-158 (1998)

11. Clarke, E.M., Jha, S., Enders, R., Filkorn, T.: Exploiting symmetry in temporal logic model checking. Formal Methods in System Design 9(1/2), 77-104 (1996)

12. Comtet, L.: Advanced Combinatorics: The Art of Finite and Infinite Expansions. D. Reidel Publishing Company (1974). URL http://books.google.com.sg/books?id=COHPgWhEssYC

13. Cormen, T.H., Leiserson, C.E., Rivest, R.L., Stein, C.: Introduction to Algorithms, Third Edition, 3rd edn. The MIT Press (2009)

14. Costa, E., Harvey, D.: Faster deterministic integer factorization. CoRR abs/1201.2116 (2012)

15. Donaldson, A.F., Miller, A.: On the constructive orbit problem. Ann. Math. Artif. Intell. 57(1), 1-35 (2009)

16. Emerson, E.A., Sistla, A.P.: Symmetry and model checking. Formal Methods in System Design 9(1/2), 105-131 (1996)

17. Goldreich, O., Micali, S., Wigderson, A.: Proofs that yield nothing but their validity and a methodology of cryptographic protocol design (extended abstract). In: FOCS, pp. 174-187 (1986). DOI 10.1109/SFCS.1986.47. URL http://doi.ieeecomputersociety.org/10.1109/SFCS.1986.47

18. Graham, R.L., Grötschel, M., Lovász, L. (eds.): Handbook of Combinatorics (Vol. 2). MIT Press, Cambridge, MA, USA (1995)

19. Hardy, G.H., Wright, E.M.: An Introduction to The Theory of Numbers, 6 edn. OUP Oxford (2008)

20. Ip, C.N., Dill, D.L.: Better verification through symmetry. Formal Methods in System Design 9(1/2), 41-75 (1996)

21. Israeli, A., Jalfon, M.: Token management schemes and random walks yield self-stabilizing mutual exclusion. In: PoDC, pp. 119-131 (1990). DOI 10.1145/93385.93409. URL http://doi.acm.org/10.1145/93385.93409

22. Kannan, R., Lipton, R.J.: Polynomial-time algorithm for the orbit problem. J. ACM 33(4), 808-821 (1986)

23. Luks, E.M.: Permutation groups and polynomial-time computation. In: DIMACS Series in Discrete Mathematics and Theoretical Computer Science, vol. 11, pp. 139-175 (1993)

24. Norman, G.: Analysing randomized distributed algorithms. In: Validation of Stochastic Systems - A Guide to Current Research, pp. 384-418 (2004)

25. Sedgewick, R., Flajolet, P.: An Introduction to the Analysis of Algorithms, 2 edn. AddisonWesley Professional (2013)

26. Shoup, V.: A Computational Introduction to Number Theory and Algebra, 2 edn. Cambridge University Press (2005)

27. Wahl, T., Donaldson, A.F.: Replication and abstraction: Symmetry in automated formal verification. Symmetry 2, 799-847 (2010)

28. Zhang, S.J., Sun, J., Sun, C., Liu, Y., Ma, J., Dong, J.S.: Constraint-based automatic symmetry detection. In: ASE, pp. 15-25 (2013) 


\section{A Completing proof of Theorem 2}

Let $\left[\begin{array}{l}n \\ k\end{array}\right]$ denote the unsigned Stirling number of the first kind, and $\left(\begin{array}{l}n \\ k\end{array}\right)$ denote $n$ choose $k$. The harmonic number $H_{n}$ is defined as

$$
H_{n}=\sum_{k=1}^{n} \frac{1}{k}
$$

In general, for an integer $s \geq 1$, the generalized harmonic number of order $s$ is defined as

$$
H_{n}^{(s)}=\sum_{k=1}^{n} \frac{1}{k^{s}}
$$

It is known that

$$
\frac{1}{n !} \sum_{k=1}^{n} k\left[\begin{array}{l}
n \\
k
\end{array}\right]=H_{n}
$$

Define

$$
f(n)=\frac{1}{n !} \sum_{k=1}^{n} k^{2}\left[\begin{array}{l}
n \\
k
\end{array}\right], \quad g(n)=\frac{1}{n !} \sum_{k=1}^{n} k^{3}\left[\begin{array}{l}
n \\
k
\end{array}\right] .
$$

It is known that

$$
\sum_{k=m}^{n}\left[\begin{array}{l}
n \\
k
\end{array}\right]\left(\begin{array}{l}
k \\
m
\end{array}\right)=\left[\begin{array}{c}
n+1 \\
m+1
\end{array}\right]
$$

(see [5]). In particular, we have

$$
\begin{aligned}
& {\left[\begin{array}{c}
n+1 \\
3
\end{array}\right]=\sum_{k=2}^{n}\left[\begin{array}{l}
n \\
k
\end{array}\right]\left(\begin{array}{l}
k \\
2
\end{array}\right)=\sum_{k=1}^{n}\left[\begin{array}{l}
n \\
k
\end{array}\right] \frac{k(k-1)}{2}=\frac{1}{2} n !\left(f(n)-H_{n}\right),} \\
& {\left[\begin{array}{c}
n+1 \\
4
\end{array}\right]=\sum_{k=3}^{n}\left[\begin{array}{l}
n \\
k
\end{array}\right]\left(\begin{array}{l}
k \\
3
\end{array}\right)=\sum_{k=1}^{n}\left[\begin{array}{l}
n \\
k
\end{array}\right] \frac{k(k-1)(k-2)}{6}=\frac{1}{6} n !\left(g(n)-3 f(n)+2 H_{n}\right) .}
\end{aligned}
$$

That is, we have

$$
\begin{gathered}
f(n)=\frac{2}{n !}\left[\begin{array}{c}
n+1 \\
3
\end{array}\right]+H_{n}, \text { and } \\
g(n)=\frac{6}{n !}\left[\begin{array}{c}
n+1 \\
4
\end{array}\right]+3 f(n)-2 H_{n}=\frac{6}{n !}\left[\begin{array}{c}
n+1 \\
4
\end{array}\right]+\frac{6}{n !}\left[\begin{array}{c}
n+1 \\
3
\end{array}\right]+H_{n} .
\end{gathered}
$$

It is known (cf. page 217 of [12]) that

$$
\begin{gathered}
\frac{1}{n !}\left[\begin{array}{c}
n+1 \\
3
\end{array}\right]=\frac{1}{2}\left(H_{n}^{2}-H_{n}^{(2)}\right) \\
\frac{1}{n !}\left[\begin{array}{c}
n+1 \\
4
\end{array}\right]=\frac{1}{6}\left(H_{n}^{3}-3 H_{n} H_{n}^{(2)}+2 H_{n}^{(3)}\right) .
\end{gathered}
$$

It is also known that $H_{n}=\gamma+\ln n, \lim _{n \rightarrow \infty} H_{n}^{(2)}=\zeta(2)=\frac{\pi^{2}}{6}$ and $\lim _{n \rightarrow \infty} H_{n}^{(3)}=\zeta(3) \approx$ 1.202 , where $\gamma \approx 0.577$ is Euler's constant and $\zeta(s)=\sum_{k=1}^{\infty} \frac{1}{k^{s}}$ is the Riemann zeta function. Hence we obtain

$$
f(n)=H_{n}^{2}-H_{n}^{(n)}+H_{n} \sim \ln ^{2} n .
$$

Putting all together, we obtain

$$
g(n)=\frac{6}{n !}\left[\begin{array}{c}
n+1 \\
4
\end{array}\right]+3 f(n)-2 H_{n} \sim \ln ^{3} n .
$$

